PHARMACOTHERAPY

\section{NAC plus nitrate therapy in $\mathrm{PCl}$}

The addition of $\mathrm{N}$-acetylcysteine (NAC) to background nitroglycerin therapy during percutaneous coronary intervention $(\mathrm{PCl})$ for ST-segment elevation myocardial infarction (STEMI) reduces infarct size and improves myocardial salvage. This finding comes from the NACIAM trial published in Circulation.

Intravenous nitroglycerin (also known as glyceryl trinitrate or GTN) is a vasodilator that can improve myocardial function in STEMI, but tolerance to high-dose nitroglycerin develops rapidly. The enzymatic process by which nitroglycerin releases nitric oxide requires sulfhydryl cofactors. NAC is a sulfhydryl-containing antioxidant that might have two potential benefits in STEMI: free-radical scavenging to minimize ischaemia-reperfusion injury, and potentiation of the haemodynamic and platelet anti-aggregative effects of nitroglycerin to improve restoration of coronary perfusion.

In the NACIAM trial, 112 patients with STEMI undergoing $\mathrm{PCl}$ and receiving background low-dose nitroglycerin $(7.2 \mathrm{mg}$ over 2 days) were randomly assigned to receive intravenous high-dose NAC $(29 \mathrm{~g}$ over 2 days) or placebo. A total of 75 patients ( 37 who received NAC and 38 who received placebo) underwent early cardiac MRI to assess infarct size. Patients assigned to NAC had an absolute $5.5 \%$ reduction in infarct size compared with placebo (median $11.0 \%$ vs $16.5 \% ; P=0.02$ ). Additionally, NAC treatment was associated with a doubling of myocardial salvage (60\%) compared with placebo $(27 \%$; $P<0.01)$, as well as more rapid resolution of chest pain.

Sivabaskari Pasupathy, lead author on the trial report, and colleagues next plan to determine the mechanisms underlying the reduction in infarct size and to evaluate the effect of NAC therapy on clinical outcomes. Future research might include "a large multicentre clinical outcome study assessing the composite end point of death and readmission with re-infarction or heart failure," adds Pasupathy.

Gregory B. Lim

ORIGINAL ARTICLE Pasupathy, S. et al. Early use of $\mathrm{N}$-acetylcysteine (NAC) with nitrate therapy in patients undergoing primary percutaneous coronary intervention for ST-segment elevation myocardial infarction reduces myocardial infarct size (the NACIAM trial). Circulation http:// dx.doi.org/10.1161/CIRCULATIONAHA.117.027575 (2017) 